\title{
Análisis del caso “Órdenes Guerra y otros vs. Chile (Fondo, Reparaciones y Costas)"
}

\author{
Case Analysis "Órdenes Guerra y otros vs Chile (Fondo, Reparaciones y \\ Costas)"
}

\author{
Sergio PEÑA NeIRA ${ }^{1}$ \\ Ignacio LAGOS RIVERA ${ }^{2}$
}

\begin{abstract}
Resumen: El presente comentario tiene por objeto el caso "Órdenes Guerra y otros" ante Corte Interamericana de Derechos Humanos, el cual versa sobre familiares de víctimas de violaciones de los Derechos Humanos cometidos durante 1973 y 1974 en Chile. Estas violaciones fueron cometidas por agentes del Estado contra víctimas de violaciones a Derechos Humanos reconocidas por el Estado de Chile en el Informe de la Comisión Nacional de Verdad y Reconciliación (8 de febrero de 1991). Los familiares de la parte lesionada, ninguno ha recibido una reparación económica por vía judicial, porque se aplicó la prescripción de la acción civil. No obstante, ellos recibieron otros beneficios administrativos por el Estado una vez restablecida la Democracia. El fundamento entregado por la Corte Interamericana de Derechos Humanos para la entrega judicial de la indemnización en su sentencia es el objeto de este comentario. A lo largo del comentario se analizará los hechos, los argumentos tanto de la parte lesionada como del Estado de Chile, los Principios Generales de Derecho y otros instrumentos jurídicos que utilizó el tribunal para determinar la Responsabilidad del Estado de Chile analizados por la Corte, y dictar sentencia.
\end{abstract}

Palabras clave: Indemnización, Derechos Humanos, Corte Interamericana de Derechos Humanos

\footnotetext{
${ }_{1}$ Profesor asociado Doctor Sergio Peña Neira, Universidad Mayor, Chile. Licenciado en Ciencias Jurídicas, Universidad de Aconcagua. Licenciado/Magíster en Relaciones Internacionales y de la UE y Administración, Universidad de Amsterdam. DEA (Magíster) Estudios Avanzados en Derecho, Universidad Internacional de Andalucía. Investigación predoctoral, Alemania, Universidad de Heidelberg, sede Santiago. Doctorado en Derecho, Universidad Internacional de Andalucía. Postdoctorado, Brandon Research Fellow, Lauterpacht Center, King's College London y U. of Cambridge. Correo electrónico: sergio.pena@umayor.cl 2 Estudiante de Derecho de la Universidad Mayor, Santiago de Chile. Ayudante de investigación en el CISS, escuela de Derecho, Universidad Mayor. El autor Ignacio Lagos Rivera dedica este comentario a Don Alejandro Rivera Arcos. Correo Electrónico: Ignacio.lagos@mayor.cl
} 
Abstract: The present commentary focuses on the judgment "Órdenes Guerra y otros contra Chile" at the Inter American Human Rights Court, ruling on the relatives of those being subject to the Human Rights violations during 1973 and 1974 in Chili. These violations were committed by agents of the State against victims of human rights already recognized by the Chilean State in the Report of the National Commission of Truth and Reconciliation (February 8,1991). None of the relatives of the injured party has obtained an economic reparation by the judiciary because it has been applied the term of the civil statute of limitations. However, they have obtained other administrative benefits by the State when Democracy was established. The foundation of the Inter American Human Rights Court to rule in favor of a judicial reparation in its sentence is the object of this commentary. Throughout the commentary evidence will be analyzed, the arguments from the injured as well as the State of Chile, General Principles of Law and other juridical instruments considered by the tribunal to define the Liability of the Chilean State analyzed by the Court in judging the case.

Keywords: Compensation, Human Rights, Inter American Human Rights Court

\section{Introducción}

La sentencia es relevante pues la Corte Interamericana de Derechos Humanos (en adelante, Corte IDH) analiza y establece la responsabilidad del Estado de Chile, respecto del caso Órdenes Guerra y otros. El conflicto jurídico consiste en haber vulnerado los “Derechos a las garantías judiciales y a la protección judicial” por el Estado de Chile y la responsabilidad en dicha vulneración. De esta manera, la Corte IDH establece la responsabilidad del Estado solucionando, asimismo el caso sometido a su jurisdicción. El objetivo expresado en la sentencia fue establecer fundamentos jurídicos para evitar la aplicación de la prescripción de la acción civil de indemnización de perjuicios por daño moral. Lo anterior en casos de Derechos Humanos conocidos por tribunales chilenos. Todos estos puntos importantes de la sentencia serán tratados en el presente comentario. Por último, en cuanto a la responsabilidad del Estado también será estudiada de manera específica en este comentario.

\section{Caso}

\subsection{PROCEDIMIENTO}

El 17 de mayo de 2017 la Comisión Interamericana de Derechos Humanos (en adelante, ComIDH) sometió el caso "María Laura Órdenes Guerra y otros" a la jurisdicción de la Corte IDH. Ello, de conformidad con lo dispuesto en los artículos 51 y 61 de la “Convención Americana de 
Derechos Humanos" (de aquí en adelante, CADH) y el artículo 35 del "Reglamento de la Corte Interamericana de Derechos Humanos".

\subsection{HECHOS}

Los familiares de los demandantes forman parte de la nómina de víctimas de violación de los Derechos Humanos reconocidas por Chile en la "Comisión Nacional de Verdad y Reconciliación". A pesar de que, los demandantes no han recibido un juicio de reparación económica por la vía judicial a pesar de ser familiares de las víctimas identificadas por el Estado. Esto, sin perjuicio, de las reparaciones por vía administrativa otorgadas por el Estado.

De aquí se desprende el hecho principal constatado por la Corte: La persistencia de la ausencia de medidas de reparación por vía Judicial derivadas del Ilícito cometido y que debe responder el Estado de $\mathrm{Chile}^{3}$. Producto de ello, retorna a los hechos originales, precisa las violaciones de los Derechos Humanos reconocidos por el Estado para resolver la controversia en torno a las reparaciones. La Corte IDH en cuanto a los hechos hace notar que existe concordancia entre el Estado y la ComIDH. Esto como consecuencia de la manifestación del Estado de Chile de aceptar los hechos probados por la ComIDH en su informe, como también los hechos, respecto de personas detenidas, secuestradas, desaparecidas o ejecutadas por agentes del Estado durante la Dictadura Militar.

\subsection{DERECHOS VULNERADOS}

Los derechos alegados son los "Derechos a las garantías judiciales y a la protección judicial" que el Estado chileno habría vulnerado. Específicamente, se vulneró el amplio contenido y alcance del "Derecho de Acceso a la Justicia”. Estos derechos están tratados particularmente en los artículos 8.1 y 25.1 de la CADH. El “Derecho a las Garantías Judiciales” está tratado en el artículo 8.1 que, como se señala en la sentencia comentada, establece:

Toda persona tiene Derecho a ser oída, con las debidas garantías y dentro de un plazo razonable, por un juez o tribunal competente, independiente e imparcial, establecido con anterioridad por la ley, en las sustanciaciones de cualquier acusación penal formulada contra ella, o para la determinación de sus derechos y obligaciones de orden civil, laboral, fiscal o de cualquier otro carácter4.

\footnotetext{
${ }^{3}$ La Corte Interamericana de Derechos Humanos precisa en la sentencia que el hecho principal es la ausencia de medidas de reparación vía judicial, debido a que, la Comisión Interamericana de Derechos Humanos se refiere a otros hechos. En virtud de esos hechos solicita ciertas medidas al Tribunal. Medidas que fueron rechazadas por el Tribunal como se verá más adelante.

${ }^{4}$ Decreto 873, 1991.
} 
Por lo tanto, la Corte IDH primeramente concluye que el Estado chileno, a través de sus tribunales, incumplió con lo dispuesto en el artículo citado. Este acto contrario a la CADH consistió en que los tribunales acogieron la prescripción civil. Al acoger dicha prescripción civil respecto de los parientes de los occisos los trataron desigualmente. Esto, por cuanto se les negó la posibilidad de conocimiento de los tribunales del fondo del asunto. Consecuencialmente, se les negó el acceso a la Justicia. Es dable indicar que dicha negación de ejercicio de la acción civil en razón de la aplicación de la prescripción indicada fue ejercida por todos los tribunales que conocieron del asunto (tribunal de primera instancia, Corte de Apelaciones y Corte Suprema).

Del mismo modo, el “Derecho a la Protección Judicial” está establecido en el artículo 25.1 de la $\mathrm{CADH}$ que establece:

Toda persona tiene Derecho a un recurso sencillo y rápido o a cualquier otro recurso efectivo ante los jueces o tribunales competentes, que la ampare contra actos que violen sus Derechos fundamentales reconocidos por la Constitución, ley o la presente Convención, aun cuando tal violación sea cometida por personas que actúen en ejercicio de sus funciones oficiales 5 .

Por ende, la Corte IDH señala que el Estado de Chile infringió también este artículo al no cumplir con garantizarles a las víctimas un procedimiento sencillo y rápido ante jueces o tribunales por actos de agentes del Estado durante la Dictadura Militar. Además, se relaciona con lo establecido en los artículos 1.1 y 2 de la misma Convención. Estos artículos son relevantes para la Corte IDH, para fundamentar la sentencia. El artículo 1.1 señala que:

Los Estados parte en esta Convención se comprometen a respetar los Derechos y Libertades reconocidos en ella y a garantizar su libre y pleno ejercicio a toda persona que esté sujeta a su jurisdicción, sin discriminación alguna por motivos de raza, color, sexo, idioma, religión, opiniones políticas o de cualquier otra índole, origen nacional o social, posición económica, nacimiento o de cualquier condición social 6 .

El artículo 2 nos señala: "Los Estados se comprometen a adoptar, a sus procedimientos Constitucionales y a las disposiciones de esta Convención, en las medidas legislativas o de otro carácter que fueren necesarias para hacer efectivos tales Derechos y libertades"7.

En ambos artículos, la Corte IDH indica la vulneración del Estado de Chile de lo dispuesto en los mencionados artículos. En primer lugar, el Estado chileno es parte de la CADH. En consecuencia, como ya se ha mencionado, se les ha negado el acceso a los demandantes por la vía judicial debido a la aplicación de la prescripción de la acción civil. Entonces, la Corte IDH

${ }^{5}$ Decreto 873, 1991.

${ }^{6}$ Decreto $873,1991$.

${ }^{7}$ Decreto 873, 1991. 
asegura que el Estado no ha adaptado a sus procedimientos las medidas necesarias para hacer efectivos tales derechos y libertades. La jurisprudencia del Tribunal ${ }^{8}$ (reiterada en el tiempo) ha estimado en situaciones de graves violaciones de Derechos Humanos, como lo ocurrido en Chile, la inadmisible e inaplicabilidad de la prescripción. Se basa la Corte IDH en lo mencionado por el "Grupo de Trabajo de las Naciones Unidas sobre las Desapariciones Forzadas o Involuntarias". Este Grupo expresó en sus observaciones generales respecto del artículo 19 de la "Declaración sobre la Protección de Todas las Personas contra las Desapariciones forzadas", que las acciones civiles de indemnización no estarán sujetas a la prescripción. También se basa en lo dispuestos en los “Principios para la Protección y la Promoción de los Derechos Humanos mediante la Lucha contra la Impunidad" establecidos por la "Comisión de Derechos Humanos de la Organización de las Naciones Unidas". Principios como como el $N^{\circ} 23$ que establece "Restricciones a la Prescripción" no son cumplidos por el Estado chileno. Tampoco cumple, conforme a la Corte IDH, con el principio $\mathrm{N}^{\circ} 32$ en cuanto a los "Procedimientos de Reparación". Para ello aplica lo dispuesto en el artículo 63.1 de la $\mathrm{CADH}$, en cuanto a que toda violación de una obligación internacional que haya producido daño implica el deber de repararlo adecuadamente dado que consagra el principio sobre "Responsabilidad del Estado". De igual manera se menciona los principios $\mathrm{N}^{\circ} 6 \mathrm{y} \mathrm{N}^{\circ} 7$ sobre la no aplicación de la prescripción de las acciones civiles si ellas tratan sobre violación de los Derechos humanos.

Llama la atención que los "hechos" son, en sede nacional, actos jurídicos procesales (sentencias) y, además, razonamiento jurídico en la aplicación de la ley chilena (declaración de la prescripción civil).

\section{Decisión de la Corte IDH}

La Corte IDH con el fin de dictar la sentencia en cuanto a las "Reparaciones" aplicó lo dispuesto en el artículo 63.1 de la CADH. El artículo 63.1 establece:

Cuando decida que hubo violación de un Derecho o Libertad protegidos en la Convención, la Corte dispondrá que se garantice al lesionado en el goce de su Derecho o Libertad conculcados. Dispondrá, asimismo, si ello fuera procedente, que se reparen las consecuencias de la medida o situación que ha configurado la vulneración de esos Derechos y el pago de una justa indemnización a la parte lesionada9 .

\footnotetext{
${ }^{8}$ La Jurisprudencia que hace mención la sentencia para dictar sentencia.

${ }^{9}$ Decreto 873, 1991.
} 
Es necesario señalar que, se deben realizar las reparaciones adecuadas del daño ocasionado por la infracción de una obligación internacional. La misma requiere la "plena restitución" la cual consiste en la restauración a la situación anterior a la violación del Derecho. El Estado de Chile infringe lo establecido en el artículo 63.1. Según la disposición, en este caso se determinó que hubo una violación de un derecho de acceso a la justicia. Los familiares de los demandantes se les privó de su libertad, fueron abusados e incluso ejecutados por agentes del Estado en el tiempo de Dictadura Militar en Chile. Chile debería, según la sentencia, haber reparado las consecuencias de la violación de Derechos Humanos con las medidas de reparación. Esto conforme a la vulneración de estos Derechos y, por consiguiente, con el pago de una justa indemnización a las partes lesionadas. Según lo dispuesto en el artículo mencionado y lo solicitado por la ComIDH y el representante de las partes lesionadas, la controversia sigue en cuanto al alcance de las reparaciones solicitadas y las planteadas por el Estado. A los demandantes no se les brindó una reparación vía judicial, por la aplicación de la prescripción de la acción civil de indemnización de perjuicios por daño moral. Entonces, el Estado nunca fue castigado, no se le impuso ninguna sanción y es ahí donde radica el conflicto.

En consecuencia, la Corte IDH procedió a dictar sentencia resolviendo la controversia planteada. En primer lugar, determinó que la parte lesionada son las víctimas declaradas en el presente caso. Las víctimas son los demandantes del caso a quienes se le privó del acceso a la justicia10 ${ }^{10}$ Luego analizó lo solicitado por la ComIDH, en cuanto a que el Estado debe adoptar las medidas de reparación pertinentes para ofrecer un recurso judicial efectivo a las víctimas. La Corte IDH analiza las sugerencias amistosas que hace el representante de las partes lesionadas. Estas solicitudes y sugerencias consisten en "Algún remedio jurídico rápido y efectivo, administrativa o de cualquier naturaleza, que dispongan las indemnizaciones que correspondan"11.

Luego la Corte IDH examina la argumentación realizada en la contestación del Estado. La misma estimo que las decisiones pronunciadas en las causas judiciales fundadas por las víctimas tienen el carácter de "Cosa Juzgada". En consecuencia, hace imposible jurídicamente restituir los procesos para dictar nuevas sentencias, por ello las reparaciones adecuadas serían una

\footnotetext{
10 Desde aquí en adelante entiéndase por "víctimas" a los demandantes del caso que fueron privados del acceso a la justicia. No hay que confundir a sus familiares víctimas de derechos humanos en la dictadura militar.

11 Corte Interamericana de Derechos Humanos, Caso Órdenes Guerra y otros vs. Chile Fondo, Reparaciones y Costas, serie C No. 372 , de 29 de noviembre de 2018 , párrafo $109^{\circ}$.
} 
"amenización monetaria"12. No obstante, en sus alegatos finales el Estado solicita a la Corte IDH las medidas de reparación sugeridas por la ComIDH y con ello, negar una compensación económica. Estás consisten en otorgarle en favor de los demandantes las medidas de reparación no patrimoniales que procedan. Ante ello la ComIDH está de acuerdo, pese a que en principio le solicita a la Corte IDH que no debe admitir los argumentos del Estado. Ello, porque, el Estado no debe invocar disposiciones de su Derecho interno para desistir de su obligación, dado que las víctimas deben contar con un recurso judicial ${ }^{13}$. Sin embargo, la Corte IDH considera lo expuesto por el Estado, debido al tiempo transcurrido y la propuesta del establecimiento de un mecanismo más expedito por el Estado. Este mecanismo debe garantizar a las víctimas las mismas reparaciones posibles de haber conseguido por la vía judicial.

Asimismo, la Corte IDH consideró la denegación de justicia surgida de una interpretación judicial contraria a la CADH. Es por esta infracción que las víctimas no han podido hacer efectivo su Derecho a reclamar y eventualmente recibir la indemnización de perjuicios vía judicial, según la Corte IDH. Por lo tanto, la medida de restitución adecuada para ese daño es disponer la garantía del acceso a un nuevo recurso judicial efectivo y rápido por el Estado para subsanar la infracción, o bien algún mecanismo alternativo a fin de cumplir con esa necesidad.

Estos son los puntos que consideró y analizó la Corte IDH. Además, el Estado, la ComIDH y el representante de los demandantes están de acuerdo. Sin embargo, la Corte IDH considera que el daño ocasionado por esta falta de acceso a la justicia es que las víctimas no han recibido la correspondiente indemnización. De tal modo que, de aceptar el argumento del Estado ello implicaría que, las víctimas quedaran sin la posibilidad de acceder a reparaciones tanto en el ámbito interno como en el internacional. Además, la Corte IDH tiene en cuenta que todos los familiares de las víctimas de este caso son personas desaparecidas o ejecutadas, y en algunos casos torturadas, hechos que son calificados como crímenes contra la humanidad.

Por consiguiente, ahora veremos la aplicación de principios para la determinación de la indemnización. La Corte IDH señala que hay que tener presente el principio de complementariedad y también el principio general del derecho de la "Cosa juzgada” en esta materia. Todo esto permite entender la razón de la Corte IDH para dictar una sentencia en la cual estima pertinente disponer el otorgamiento por el Estado de una compensación directa a cada

\footnotetext{
${ }^{12}$ Amenizar significa hacer agradable y entretenido una situación, por lo tanto, una Amenización Monetaria sería hacer agradable una situación monetariamente.

${ }^{13}$ La doctrina está conteste con esta afirmación de la Corte IDH.
} 
uno de los demandantes del caso. Es importante destacar el análisis de la Corte IDH sobre la jurisprudencia de la Corte Suprema chilena en los últimos años en este tipo de casos. En estos casos los montos indemnizatorios por perjuicios morales oscilan entre los treinta millones y ciento cincuenta millones de pesos chilenos que la jurisprudencia nacional ha otorgado a cada uno de los familiares de las víctimas. Estos montos son independientes del tipo de filiación (entendida como "dependencia que tiene algunas personas o cosas respecto de otra u otras principales") de estos con la víctima desaparecida o ejecutada. Por lo tanto, en aplicación al principio de complementariedad la Corte IDH estima pertinente fijar la cantidad total que se expondrá en el título de "Responsabilidad del Estado". Por ende, en base a lo analizado en la sentencia dictada por la Corte IDH se ha aplicado correctamente el Derecho Internacional como por sus principios a los hechos en controversia.

Es destacable otra correcta decisión de la Corte IDH, en cuanto a las otras medidas solicitadas, específicamente no repetición. Una de estas medidas, es la dictada por ComIDH la cual es recomendada al Estado chileno y solicitada a la Corte IDH. Esta medida consiste en que, el Estado chileno debe adoptar medidas administrativas y de cualquier otra índole, con el objeto de adecuar tanto la legislación como las prácticas judiciales chilenas a la prohibición de prescripción a acciones civiles que busquen reparación de perjuicios. Esto debido a que la ComIDH considera que el Estado no ha adoptado una solución general y duradera para cumplir con estas medidas. El fundamento de lo anterior es el solo cambio de la jurisprudencia de la Corte Suprema de Chile a favor de la no aplicación de la prescripción en casos similares al analizado. Si bien la ComIDH lo considera insuficiente, la Corte IDH le responde diciendo que la infracción de los Derechos Humanos en estos casos, no se originan en disposiciones específicas del Derecho interno chileno, sino en la interpretación que del mismo se hicieron. Además, lo hace en atención a la buena fe del Estado en el cumplimiento de sus obligaciones.

\section{Responsabilidad del Estado}

El concepto de "responsabilidad internacional" es un concepto complejo. El Estado chileno generó un daño al no brindarles soluciones por vía judicial a las partes demandantes como consecuencia de la aplicación de la prescripción. Complementando lo anterior, es importante analizar el concepto de Responsabilidad. Es dable destacar la precisión, a nuestro parecer perfecta, que hace la Corte IDH, en cuanto, a la extensión de la responsabilidad. A fin de entender, 
es necesario hacer un análisis de los antecedentes que consideró la Corte IDH para dictar la sentencia. Este tema lo analizaremos en tres partes:

1. En primer lugar, es necesario analizar los tratados en los que es parte el Estado de Chile. Entonces, hay que analizar la CADH de la cual el Estado de Chile forma parte. De la misma, la Corte IDH cita una serie de artículos para determinar y dictar sentencia, así los artículos 8.1 respecto al "Derecho a las Garantías Judiciales”, el 25.1 respecto del "Derecho a la Protección Judicial” y el artículo 63.1 respecto de las facultades de la corte. Estos artículos son alegados por los demandantes, afirman que han sido conculcados por el Estado de Chile, lo cual es ratificado por la Corte IDH. Además, los demandantes arguyen la transgresión de los artículos 1.1 y 2 de la misma Convención. Estos artículos versan sobre el respeto a los derechos y libertades reconocidas en la Convención y a garantizar su libre y pleno ejercicio a todas las personas. También hablan respecto al compromiso del Estado en adoptar los procedimientos o medidas que fueren necesarias para hacer efectivos tales derechos y libertades.

El Estado de Chile no cumple lo establecido en dichos artículos conforme al razonamiento que expondremos. Éste vulneró los derechos de acceso a la justicia, al no respetar los derechos y libertades de la Convención. El Estado tampoco adoptó los procedimientos necesarios para otorgar soluciones efectivas a la vulneración de los derechos y libertades. El Estado de Chile debiese cambiar la jurisprudencia de sus tribunales en el tiempo en que se realizaron los daños, según la Corte IDH. De esa manera sus órganos, les hubiesen otorgado una solución eficaz a los demandantes para poder sanear los daños causados. Por ello, la Corte IDH aplicó el artículo 63.1 de la misma $\mathrm{CADH}$, artículo que regula sobre reparaciones. Este artículo 63.1 le otorga la jurisdicción a la Corte IDH para garantizarles a los lesionados el goce de sus derechos conculcados por el Estado de Chile. La Corte IDH determinó que el Estado de Chile violó los derechos establecidos en la Convención, específicamente los de los artículos 8.1 y 25.1 que versan sobre los derechos y libertades de acceso a la justicia y determina la responsabilidad del Estado chileno en el incumplimiento de sus obligaciones.

Sin embargo, antes de señalar las reparaciones y sanciones que deberá asumir el Estado de Chile, la Corte IDH hace ciertas precisiones como: a.- Que no ordenará al Estado de Chile, revivir los procesos ya fenecidos, porque, respeta el "Efecto de Cosa Juzgada” Así mismo b.- Tampoco obligará al Estado chileno a modificar su Código Civil por considerar que el error nace de una interpretación de los artículos, no de lo establecido en ellos. De esta manera la Corte IDH busca respetar la autonomía del país como también el principio de "Cosa Juzgada". 
Finalmente, en conformidad con el artículo 63.1 la Corte IDH resuelve que el Estado de Chile es responsable de los daños causados a las víctimas ${ }^{14}$ del caso. De esta forma obliga al Estado al pago de una justa indemnización a la parte lesionada. La Corte IDH resuelve de esta manera, porque, el artículo 63.1 faculta a la corte ordenar la reparación las consecuencias causadas por la vulneración a estos derechos a través de la indemnización. Asimismo, estudia las indemnizaciones otorgadas por la Corte Suprema de Chile en casos similares aplicando el “Principio de Complementariedad" para que la sanción sea más justa para ambas partes.

2. En segundo lugar, se procederá a analizar las normas del Derecho de la Costumbre Internacional y Nacional, con relación a la responsabilidad. Lo interesante es que la Corte IDH analizó la jurisprudencia de la Corte Suprema de Chile en cuanto a las indemnizaciones otorgadas en fallos similares al que se está analizando. Debido al análisis realizado por la Corte IDH respecto de la jurisprudencia sostenida en estos últimos años por la Corte Suprema se percibe una importante precisión, en cuanto a la "Extensión de la Responsabilidad" del Estado de Chile. La Corte IDH analizó la costumbre para fijar las indemnizaciones en este tipo de casos. De esta manera, la Corte IDH decide fijar la indemnización que debe pagar el Estado en US $\$ 180.000,00$ a cada uno de los afectados. Así estaría acorde con las indemnizaciones fijadas por la Corte Suprema de Chile. El monto es acorde a la costumbre en casos similares fallados por la Corte Suprema y, además, obliga al Estado a pagar la indemnización a las personas cuyos derechos fueron vulnerados. La Corte IDH asimiló los actos nacionales de entrega de indemnización como opinio juris y, al ser reiterados, cumplen el requisito de actos materiales generando una costumbre nacional, y en esta sentencia, internacional. La Corte IDH considera a la condena de indemnización como un hecho.

3. En el tercer y último punto, se analizará los Principios Generales del Derecho que la Corte IDH consideró al momento de dictar sentencia. La Corte IDH consideró los "Principios para la Protección y la Promoción de los Derechos Humanos mediante la Lucha contra la Impunidad" generados por la Comisión de Derechos Humanos de la Organización de las Naciones Unidas. Específicamente en ellos el principio 23 respecto a las "Restricciones a la Prescripción" y el principio 32 en cuanto a los “Procedimientos de Reparación” y los Principios 6 y 7 que versan en cuanto a la no aplicación de la prescripción de las acciones civiles.

${ }^{14}$ Hay que recordar que las víctimas son los demandantes del caso a quienes se les privó del acceso a la justicia. 
Estos principios son esenciales para entender la decisión del Corte IDH. Ello, debido a que, el primer principio mencionado se refiere a la protección y promoción de los Derechos Humanos para evitar la impunidad. Los tribunales chilenos no cumplieron con este principio al no concederles a las víctimas del caso acceso a la justicia, ello como consecuencia de la aplicación de la prescripción de sus acciones civiles. Lo anterior nos lleva a entender los otros principios, como lo son los principios 23 en cuanto a las restricciones a la prescripción y los principios 6 y 7 que versan sobre la no aplicación de la prescripción de las acciones civiles. Estos principios son adoptados por la Corte IDH. En caso de graves violaciones a los Derechos Humanos los principios hacen inadmisible e inaplicable la prescripción. Entonces, están protegidos y garantizados estos derechos en la CADH como en los Principios Generales del Derecho. Sin embargo, los tribunales chilenos hicieron una mala interpretación de los casos y aplicaron la prescripción.

En cuanto a los procedimientos de reparación, principio 32, expresamente indicado por la sentencia, se generó un daño a los demandantes por su falta de aplicación. De esta manera, los daños causados deben ser reparados por el Estado. Ello justificaría la decisión del tribunal de aplicar el artículo 63.1 de la CADH otorgándole la competencia a la Corte IDH. Esta competencia permite disponer que el Estado de Chile garantice los derechos y libertades conculcados a los demandantes. Con ello también obliga al Estado a pagar una justa indemnización a la parte lesionada.

De esta manera, se proseguirá a analizar los siguientes principios deducidos por la Corte IDH los cuales fueron cruciales para determinar el fallo. El primero es el "Principio de Complementariedad", el cual es de suma importancia, porque, es aplicado por la Corte IDH para poder emplear la jurisprudencia de la Corte Suprema de Chile. Así se fija el monto de la Indemnización a pagar. Esto es una decisión correcta de la Corte IDH. La aplicación de este principio permite establecer la "Extensión de Responsabilidad" precisa para el Estado de Chile en cuanto a la indemnización a pagar. El segundo principio deducido por la Corte IDH es el Principio de "Cosa juzgada" o el "Efecto de Cosa Juzgada”. Este principio, al igual que el anterior tiene suma importancia. Sirven a la Corte IDH en descartar solicitudes similares tanto de la ComIDH como del representante de las partes lesionadas. Las mismas consisten en volver a revivir los procesos en los cuales ya se dictó sentencia. Además, el Estado de Chile le informa a la corte que estos procesos ya fueron resueltos con "Efecto de Cosa Juzgada", en consecuencia, ya no se puede volver a tratar por ningún tribunal. La Corte IDH al decidir respetar la institución 
de cosa juzgada lo hace al ser parte de los Principios Generales del Derecho. De esta manera el tribunal le ordena al Estado establecer un mecanismo más expedito, el cual garantice a las víctimas las mismas reparaciones alcanzables por la vía judicial. En conformidad con el principio anterior, se puede inferir el principio de la "Autonomía de la Voluntad". Si bien no lo dice explícitamente la Corte IDH, es posible inferirla debido a como se expresa da a entender que respeta la autonomía de la voluntad del Estado de Chile. Ello se ve reflejado al no obligar al Estado a realizar medidas o actuaciones no acordadas en los tratados. Como sabemos la CADH es un tratado, por ende, es deber de las partes, respetar la voluntad del tratado, que como sabemos la voluntad es uno de los requisitos de existencia del tratado. Igualmente se refleja al rechazar la solicitud de la ComIDH, que consistía en que el Estado chileno debía modificar su Código Civil. Es aquí donde el tribunal hace un correcto análisis al determinar que las infracciones de los Derechos Humanos no se originan en disposiciones específicas del Código Civil chileno. Las infracciones de los Derechos humanos se originan en la interpretación que del mismo se hicieron. Además, el tribunal menciona que la Corte Suprema de Chile ya había modificado su jurisprudencia respecto a casos similares como los alegados en la Corte IDH y, por lo tanto, no venía al caso sancionar y solicitarle al Estado de Chile tal medida de reparación.

Es por estos tres puntos: a) los tratados de los que forma parte el sujeto de Derecho Internacional, b) las normas del Derecho de la Costumbre y c) los Principios Generales del Derecho que consideramos que la decisión de la Corte IDH como correcta y precisa, porque, establece una “Extensión de Responsabilidad" precisa, acorde a todo lo anteriormente analizado y que no resulta abusiva hacer cumplir al Estado de Chile.

\section{Efectos en la Jurisprudencia chilena: Interpretación judicial}

En el desarrollo y aplicación del caso descrito encontramos una sentencia que ha venido a aplicar el razonamiento expresado en el caso. En los párrafos vigésimo noveno y siguientes de la sentencia caratulada "Parvez/Fisco de Chile (Subsecretaría prevención del delito)" rol C-11847201815, donde se cita el caso ya tantas veces mencionado. En el párrafo vigésimo noveno se refiere al caso como de "responsabilidad" por violación de los derechos a las "garantías y protección judiciales. Lo expresado como consecuencia de la aplicación de la figura de prescripción a acciones civiles de reparación relacionadas con "Crímenes de lesa humanidad.".

${ }^{15}$ Cuarto Juzgado Civil de Santiago, Rol No. 11847 de 20 de febrero de 2019. 
Luego en el considerando trigésimo se hace referencia al reconocimiento del Estado en aceptar "conclusiones y consecuencias jurídicas" del "Informe del Fondo adoptado por la Corte Interamericana de Derechos humanos"16. Sirve de fundamento al rechazo a la prescripción extintiva $^{17}$ y se reconoce en el considerando trigésimo séptimo la indemnización correspondiente ${ }^{18}$.

\section{Normas jurídicas citadas}

Decreto 104, sobre Promulga el Estatuto de Roma de la Corte Penal Internacional. Diario Oficial, 1 de agosto de 2009.

Decreto 873, sobre San José de Costa Rica. Diario Oficial, 5 de enero de 1991.

Decreto 809, sobre Convención Interamericana para prevenir y sancionar la tortura, 26 de noviembre de 1988.

\section{Jurisprudencia citada}

Parvex con Fisco de Chile [Subsecretaría Prevención del Delito]: 4 Juzgado Civil de Santiago [acción de indemnización de perjuicios], 20 de febrero de 2019. [Disponible en: https://bit.ly/3x5HIXq]. [Fecha de consulta: 05 de junio de 2020].

${ }^{16}$ Cuarto Juzgado Civil de Santiago, Rol No. 11847 de 20 de febrero de 2019, considerando vigésimo noveno y considerando trigésimo.

${ }^{17}$ Cuarto Juzgado Civil de Santiago, Rol No. 11847 de 20 de febrero de 2019 considerando trigésimo segundo.

${ }^{18}$ Cuarto Juzgado Civil de Santiago, Rol No. 11847 de 20 de febrero de 2019 considerando trigésimo séptimo. 